\title{
RESEARCH PROBLEMS
}

\section{R. Bellman: Probability theory.}

Consider the recurrence relation $x_{n+1}=a x_{n}-b x_{n}^{2}+z_{n}, x_{0}=c$, where $a$ and $b$ are given parameters and the $z_{k}$ constitute a set of random variables drawn from a common distribution. Determine the asymptotic behavior of $E\left(x_{n}\right)$ under various assumptions concerning $a, b$ and the distribution of the $z_{k}$. (Received February 27, 1956.)

\section{R. Bellman: Number theory.}

The relation $\sum_{k \leqq N} 2^{\gamma(k)} \sim N \log N$ (an analogue of a classical device of S. Bernstein in probability theory), where $\gamma(k)$ represents the number of prime divisors of $k$, can be used to show that the number of numbers less than $N$ which have more than $c \log \log N$ prime divisors is $O\left(N /(\log N)^{\text {clog2}}\right)$ as $N \rightarrow \infty$. Can one substantially improve this estimate for large $c$ ? (Received February 27, 1956.)

\section{A. D. Wallace: $A$ problem on minimax semi-groups.}

Let $M_{n}$ be the set of all $n \times n$ matrices whose entries lie in the closed unit interval and define a multiplication in $M_{n}$ by $(\alpha, \beta)_{i j}=\operatorname{Max}_{k}\left(\operatorname{Min}\left(\alpha_{i k}, \beta_{k j}\right)\right.$ ). Clearly $M_{n}$ is a topological semi-group which is homeomorphic with the $n^{2}$-cell. If $S$ is a topological semi-group which is homeomorphic with an $n^{2}$-cell is it possible to give an abstract description of $S$ that will identify it with $M_{n}$ ? It would be useful to have such a description even at the expense of supplying $S$ with a dual operation such as obviously exists in $M_{n}$. (Received April 27, 1956.) 